\title{
Applications of Math Microscope in the Event Horizon Telescope
}

\author{
Evgeni Nikolaevich Terentiev", *, Nikolay Evgenyevich Shilin-Terentyev ${ }^{2}$ \\ ${ }^{1}$ Department Mathematical Modelling and Informatics, Faculty of Physics, M. V. Lomonosov Moscow State University, Moscow, Russia \\ ${ }^{2}$ EPAM Systems, Moscow, Russia
}

Email address:

en.teren@physics.msu.ru (E. N. Terentiev), nikolay.terentyev@gmail.com (N. E. Shilin-Terentyev)

${ }^{*}$ Corresponding author

\section{To cite this article:}

Evgeni Nikolaevich Terentiev, Nikolay Evgenyevich Shilin-Terentyev. Applications of Math Microscope in the Event Horizon Telescope. International Journal of Astrophysics and Space Science. Vol. 9, No. 2, 2021, pp. 21-31. doi: 10.11648/j.ijass.20210902.11

Received: October 18, 2020; Accepted: November 7, 2020; Published: June 30, 2021

\begin{abstract}
The paper describes the insides - the basic concepts of the Math Microscope, demonstrates the results of Super-Resolution images obtained from the Event Horizon Telescope and analyzes the results of the movement of clusters of stars that go around the Black Hole. The presence of point objects - single stars in the SR image allowed us to implement a new breakthrough approach in the problem of SR images of Powehi Black Hole in the concept of MM. In the paper, we reviewed and illustrated new concepts: Invertability Indicators and Adequacy Characteristics of discrete Models of Apparatus Functions. With these new concepts, in the inverse problem, for the first time, we were able to answer simple questions: What are we dealing with? Moreover, have we solved the inverse problem? The paper demonstrates the "manual solution" of the problem of Reconstruction of AFs and Super-Resolution on MM. In the Discussion at the end of the paper, we pose the problem of creating two Artificial Intelligences for the automated solution of the R\&SR problem with the interpretation of the SR results of BH images from EHT.
\end{abstract}

Keywords: Super-Resolution, Conditionality, Apodization in Inevitability, Modulation Transfer Function, Convolution, Fourier Transform

\section{Introduction}

Modern methods (such as regularization) for solving inverse problems are fundamentally ineffective, because they assume "a priori smoothness of solutions" [1]. We proceed from "a priori non-smoothness of solutions" solutions consist of their points (in our case, these are clusters of stars). Point objects allow us to Reconstruct Apparatus Functions (AF) or Antenna Pattern (AP) of the Event Horizon Telescope (EHT).

The identification of points - clusters of stars with the estimation of the values of the achieved Super Resolutions is the basis of our methods.

We emphasize that the methods based on the Lagrangian formalism as in regularization have little to do with the methods of discrete mathematics with their own peculiarities.

Methods of Reconstruction and Super Resolution in Mathematical Microscope (R\&SR in MM) of objects are intended for the Intelligent Analysis (IA) of data on objects observed through measuring devices whose AF or AP are not defined, are known with errors and even when they are precisely known. The methods of R\&SR objects are based on the (Apodization search or) Reconstruction of mathematical models of discrete reversible AF $\mathrm{O}$ with minimal $\operatorname{Nor}(\mathrm{R}), \mathrm{R}=\mathrm{O}^{-1}$. Conditionality is the main setting of (AF) $\mathrm{O}$ to increase resolution as result of focusing MM. The conditionality is numerically equal to the reciprocal of the minimum value of Modulation Transfer Function (MTF) $\mid(\mathrm{M}(\mathrm{O}) \mid$ or the magnitude of this gap - $\mid(\mathrm{M}(\mathrm{pO}) \mid$. We introduce the magnitude $\mathrm{SR}$ of the estimating Super-Resolution [1-3]. The concept of the MM we applied for an image from the EHT.

\section{Basic Concepts, Insides of the MM}

\subsection{Main problem, Reconstruction of $A F$}

With the Conditioned SR (CSR), we will associate the problem of choosing a working discrete AF model $\mathrm{O}$ with an 
invertible $\mathrm{R}=\mathrm{O}^{-1}$ and a small inverse norm $\operatorname{Nor}(\mathrm{R})=\|\mathrm{R}\|$. If $\operatorname{Nor}(\mathrm{R})$ is large, then we are forced to reduce $\operatorname{Nor}(\mathrm{pR})$ by increasing the conditionality parameter DI (from word DIAPAZON) to obtain acceptable Nor(pR).

Note that $\operatorname{Nor}(\mathrm{pR})$ is the response to noise (or is there a standard deviation $\sigma$ gain of white noise, $\operatorname{Nor}(\mathrm{pR}) * \sigma)$, which determine the accuracy of solving the inversion problem in the presence of additive white noise $\sigma$.

The task of choosing the conditioned $\mathrm{AF} \mathrm{pO}=\mathrm{pR}^{-1}$ is posed as a minimum problem (maximum accuracy) $[2,3]$ :

$$
\min _{\mathrm{LO}}\{\|\mathrm{pR}\| \mid \operatorname{Err}(\mathrm{pO}) \leq \mathrm{err}\}, \quad \mathrm{LO}=\{\mathrm{pO} \mid[\mathrm{Loc}, \mathrm{SDx}, \mathrm{DI}]\},
$$

Loc is the integer length of the domain of definition, taking into account the addition of one to zero. If the grid is fine, then Ste is an array of digitizing steps. The transition to an image on a coarse grid is obtained for ste $>1$ from array Ste. In addition, if we have a regular pixel image, then we can interpolate the $\mathrm{AF} \mathrm{O}$ and the image at a smaller step $\mathrm{dx}<1$ from array Dx.

\subsection{The Characteristics Adequacy of AF Model}

The Characteristics Adequacy (CAM) of the discrete AF Model $\mathrm{pO}[2,3]$ is:

$$
\mathrm{CAM} \mathrm{pO}=\mathrm{TAB}\{\mathrm{x}=\operatorname{Nor}(\mathrm{pR}), \mathrm{y}=\operatorname{Err}(\mathrm{pO}), \mathrm{z}=\mathrm{II}(\mathrm{pR} * \mathrm{O})\} \text {. }
$$

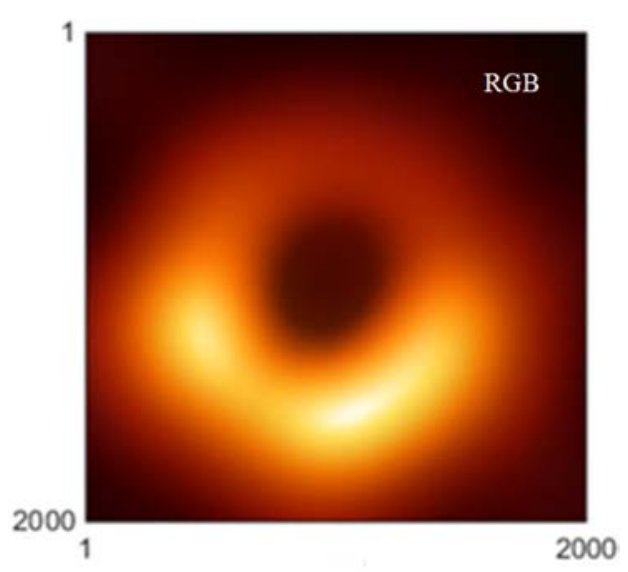

(a)

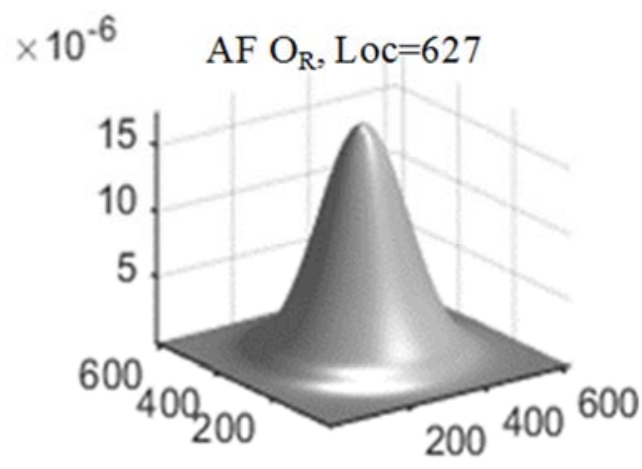

(b)

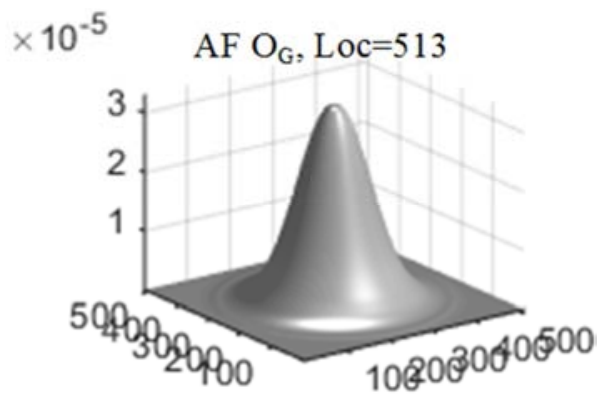

(c)

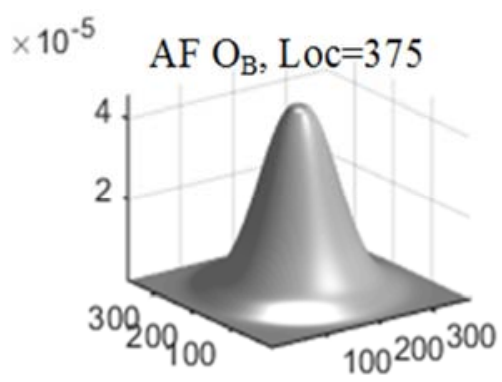

(d)

Figure 1. (a) there is a fragment of the BH image obtained with EHT on a full grid with ste $=1,(b-d)$ there are reconstructed $A F O_{R}, O_{G}$ and $O_{B}$.

$\mathrm{Iz}=10^{-13}$ is the mantissa error (or Instrumental zero.) in calculating the Fourier coefficients. In connection with this Iz restriction, we consider the $\mathrm{CAM} \mathrm{AF} \mathrm{zO}$ variant, taking into account Iz the inversion of the two-dimensional MTF M(O) (in the record without indices):

$$
\mathrm{M}(\mathrm{zR})=\left\{\begin{array}{l}
1 / \mathrm{M}(\mathrm{O}), \text { for all }|\mathrm{M}(\mathrm{O})|>\mathrm{Iz} \\
\mathrm{M}(\mathrm{O})
\end{array}\right.
$$

$$
\mathrm{CAM} z \mathrm{z}=\mathrm{TAB}\{\mathrm{x}=\operatorname{Nor}(\mathrm{zR}), \mathrm{y}=\mathrm{Err}(\mathrm{zO}), \mathrm{z}=\mathrm{II}(\mathrm{zR} * \mathrm{O})\}
$$

Note that the most conditionality object $(\mathrm{DI}=1)$ in mathematics is the Delta Kronecker symbol $\mathrm{DK}=\mathrm{DK}(\mathrm{i}, \mathrm{j})=\{$ if $\mathrm{i}=0$ and $\mathrm{j}=0,1$ else 0$\}$, MTF $\mathrm{M}(\mathrm{DK})==1$ - identically equal to 1.

For ideal devices with $\mathrm{AF} \mathrm{O}=\mathrm{DK}$, the formulation of problems of the type (1) "It is not necessary to construct MM to increase SR".

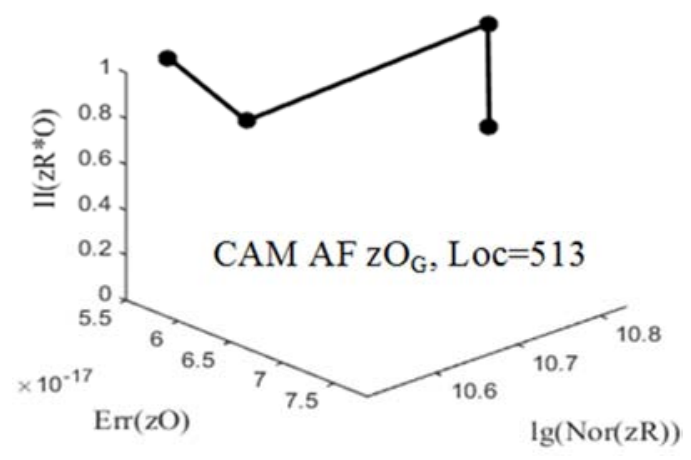

(a) 


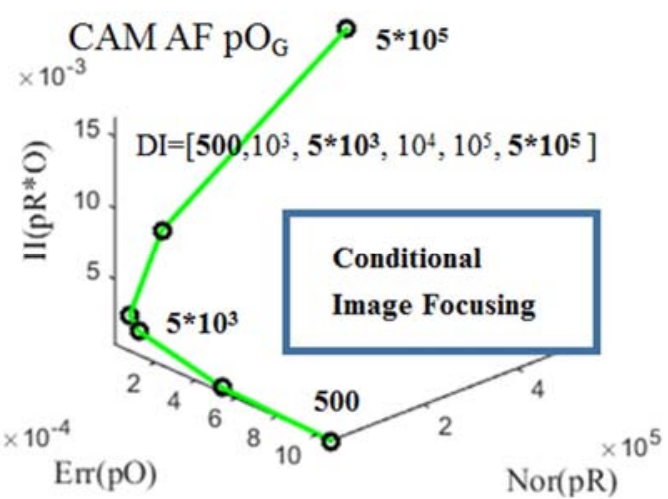

(b)
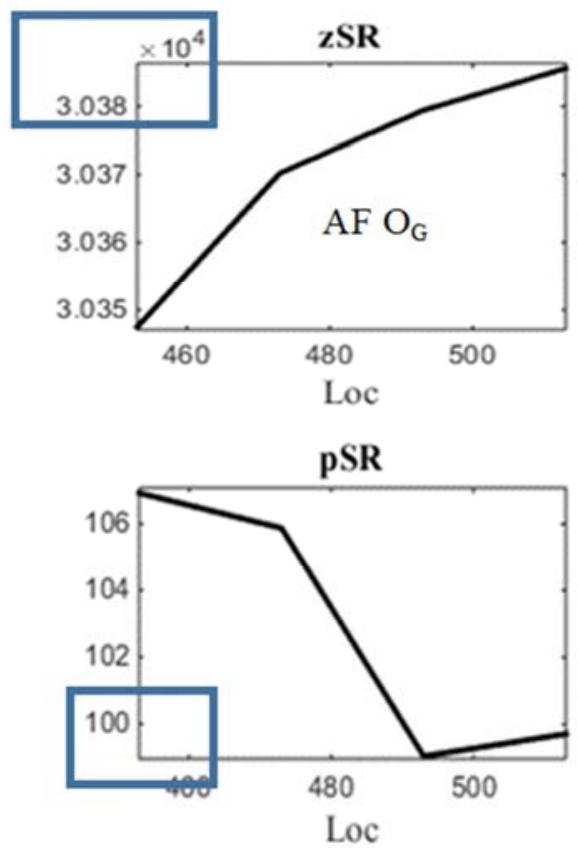

(c)

Figure 2. (a) there is CAM AF zO⿰亻⿱口木g, (b)- CAM AF pO, (c) - zSR values $\sim 30000$ are down by conditionality $D I=5000$ to $p S R \sim 100$.

\subsection{Indicator of Invertability}

In the method of regularization of Academician Tikhonov A. N. [1], the asymptotic statement connects the regularized approximate solution and the exact solution with the clause "if it (exact solution!) exists". We suggest using Indicator of Invertability (II) in addition of such statement.

Theorem [3, 4]: If the value of the II $\mathrm{II}(\mathrm{zR} * \mathrm{O})=\mathrm{zR} * \mathrm{O}(0,0)=1$, then there is an inversion $\mathrm{zR}=\mathrm{R}=\mathrm{O}^{-1}$. If $\mathrm{II}(\mathrm{zR} * \mathrm{O})<1$, then we have incomplete Iz inversion of AF O to $\mathrm{zR}$.

Note that if conditionality $\mathrm{DI}=(\mathrm{Iz})^{-1}=10^{13}$, then in the reversible case $\mathrm{II}(\mathrm{zR} * \mathrm{O})=1 \mathrm{CAM} \mathrm{pO}=\mathrm{CAM} \mathrm{zO}(2,4)$ are those same.

\subsection{Super-Resolution Function, SR Values}

If there is a normalization of $\mathrm{AF} \mathrm{O}: \sum \mathrm{O}=1$, and at zero MTF $\mathrm{M}(\mathrm{O})(0,0)=1$, then we will evaluate the super-resolution caused by the inversion of Iz by SR functions depending on three parameters in $\mathrm{LO}(1)$ :

$$
\begin{gathered}
\mathrm{pSR}=\sum \mathrm{M}(\mathrm{pR}) * \mathrm{M}(\mathrm{O}) / \sum \mathrm{M}(\mathrm{O}), \\
\mathrm{zSR}=\sum \mathrm{M}(\mathrm{zR}) * \mathrm{M}(\mathrm{O}) / \sum \mathrm{M}(\mathrm{O})
\end{gathered}
$$

Note that $\mathrm{SR} \geq 1$. If $\mathrm{AF} \mathrm{O}=\mathrm{DK}$, then $\mathrm{SR}=1$. In reversible cases, SR is a function with saturation by two parameters: the length of the domain of definition Loc and the conditionality DI, see Figure 2 (c).

Let us explain: The SR value is the ratio of the area of the "AF O spot" to the area of the smaller "AF $\mathrm{pR} * \mathrm{O}$ spot" in terms of the MTFs (5) in the saturated case.

In addition, the maximal value $\mathrm{SR}$ is equal to $\mathrm{zSR}$ or simply is the "area of the AF O spot", measured in pixels (5)!

\section{5. $\mathrm{AF} \mathrm{pO}$ and zO Errors}

The continuous parametric AF parO model, we associate the discrete AF $\mathrm{O}$ with the calculation (direct transformation) of the MTF M (O). If we build an inverse AF according to $\mathrm{M}(\mathrm{O})$, then we get the result of the $\mathrm{AF} \mathrm{zO}$ with the Instrumental (mantissa) error Iz. The conditionality adjustment according to DI is that small values of $\mathrm{M}=\mathrm{M}(\mathrm{O})$ move away from $\mathrm{Iz}$ by values $1 / \mathrm{DI}>\min |\mathrm{M}(\mathrm{O})|$ with the production of MTF $\mathrm{pM}$. We realize the inverse transformation from MTF pM to AF pO [3]. Errors $\operatorname{Err}(\mathrm{pO}), \operatorname{Err}(\mathrm{zO})$ we estimated by the formulas [3]:

$$
\begin{aligned}
\operatorname{Err}(\mathrm{pO}) & =\mathrm{SD}(\mathrm{O}-\mathrm{pO}) / \max (\mathrm{O}), \\
\mathrm{SD}(\mathrm{O}-\mathrm{pO}) & =\operatorname{sqrt}\left(\sum(\mathrm{O}-\mathrm{pO})^{2} /\left(\mathrm{Loc}^{2}-1\right)\right.
\end{aligned}
$$

and analogically $\operatorname{Err}(\mathrm{zO})$.

\section{Illustrations of Basic Concepts, Insides of the MM}

\subsection{Illustrations of Basic Concepts, Insides of the MM}

The initial data are presented on coarse grids with the ste $=10$ and $\mathrm{ste}=5, \mathrm{SR}$ is implemented with the conditionality $\mathrm{DI}=10^{4}$ and $5 * 10^{4}$.

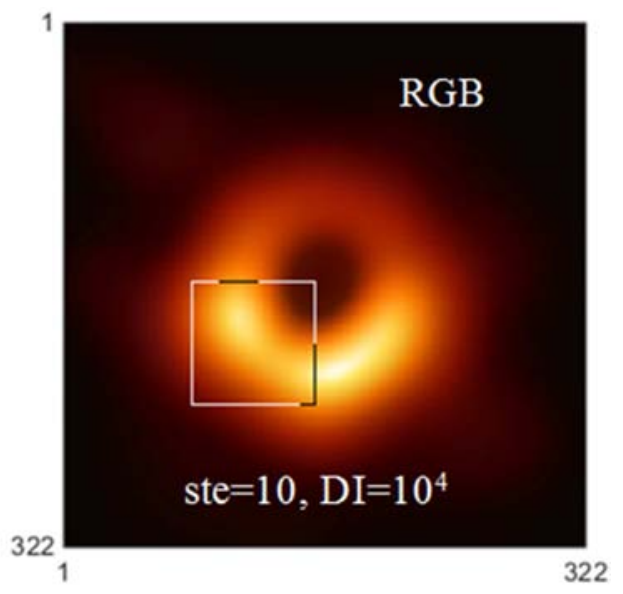



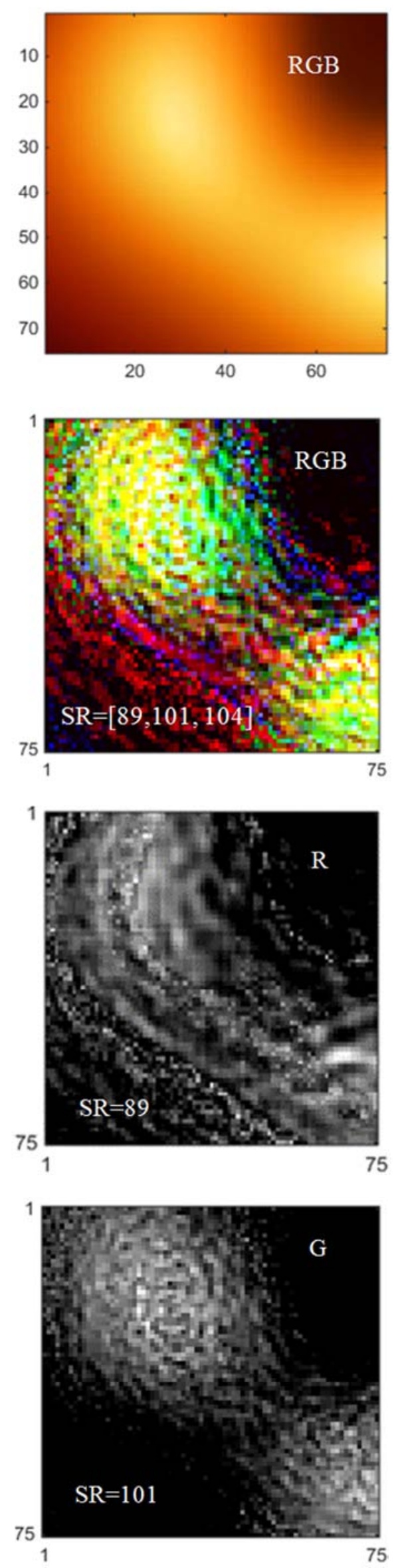

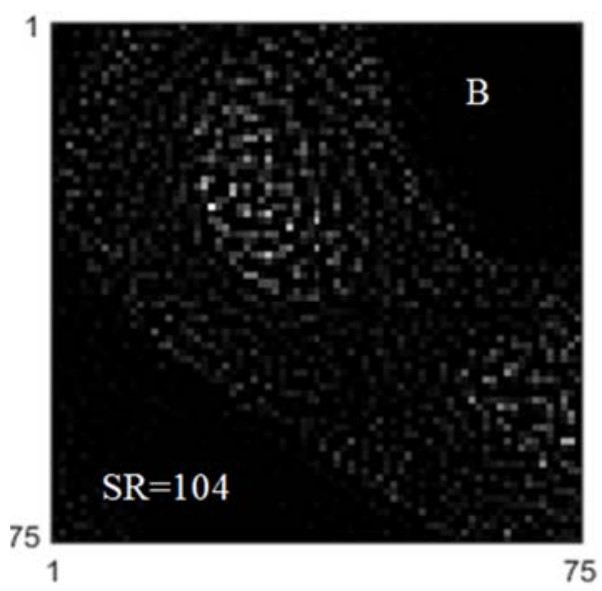

Figure 3. Coarse grid with the ste $=10, D I=10^{4}$.
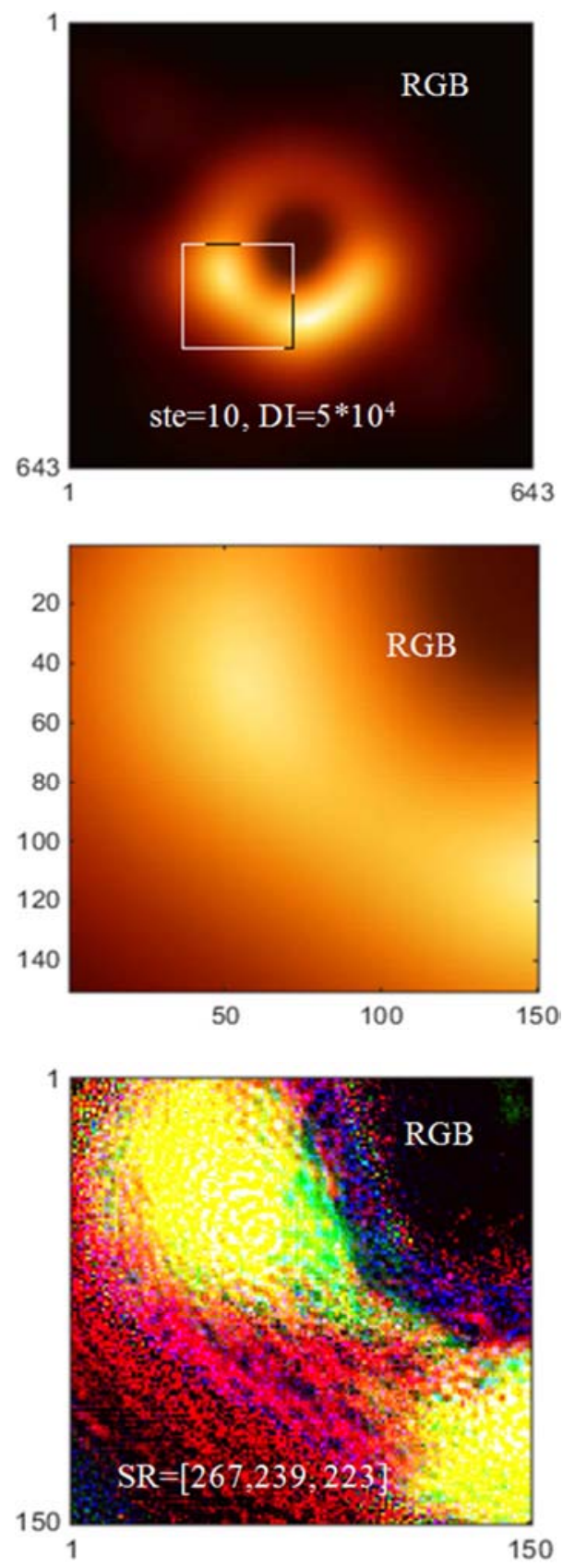

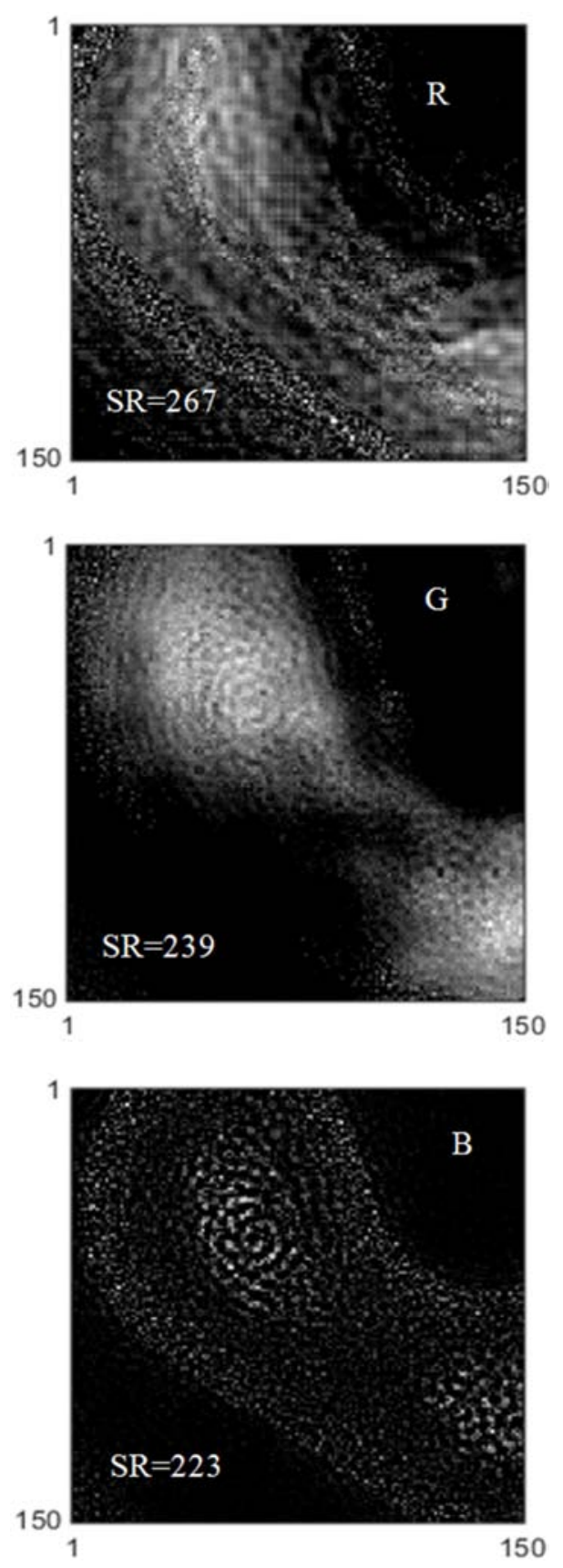

Figure 4. Coarse grid with the ste $=5, D I=5 * 10^{4}$.

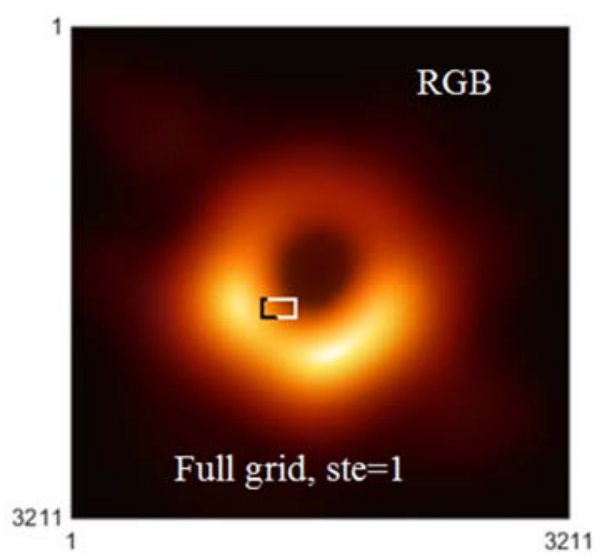

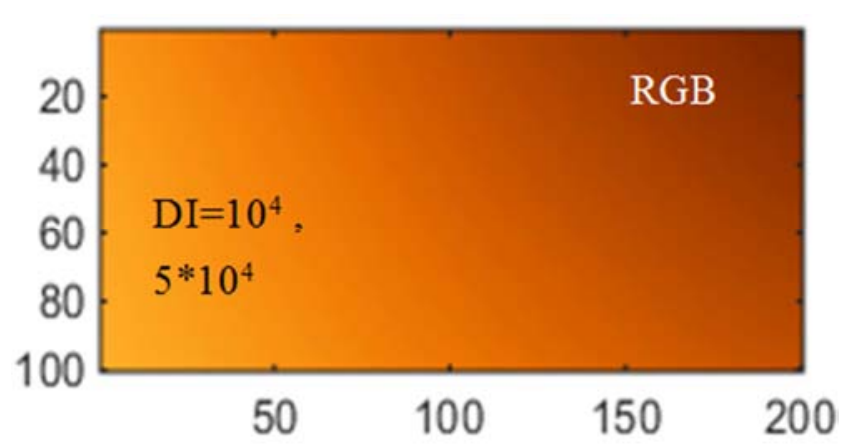
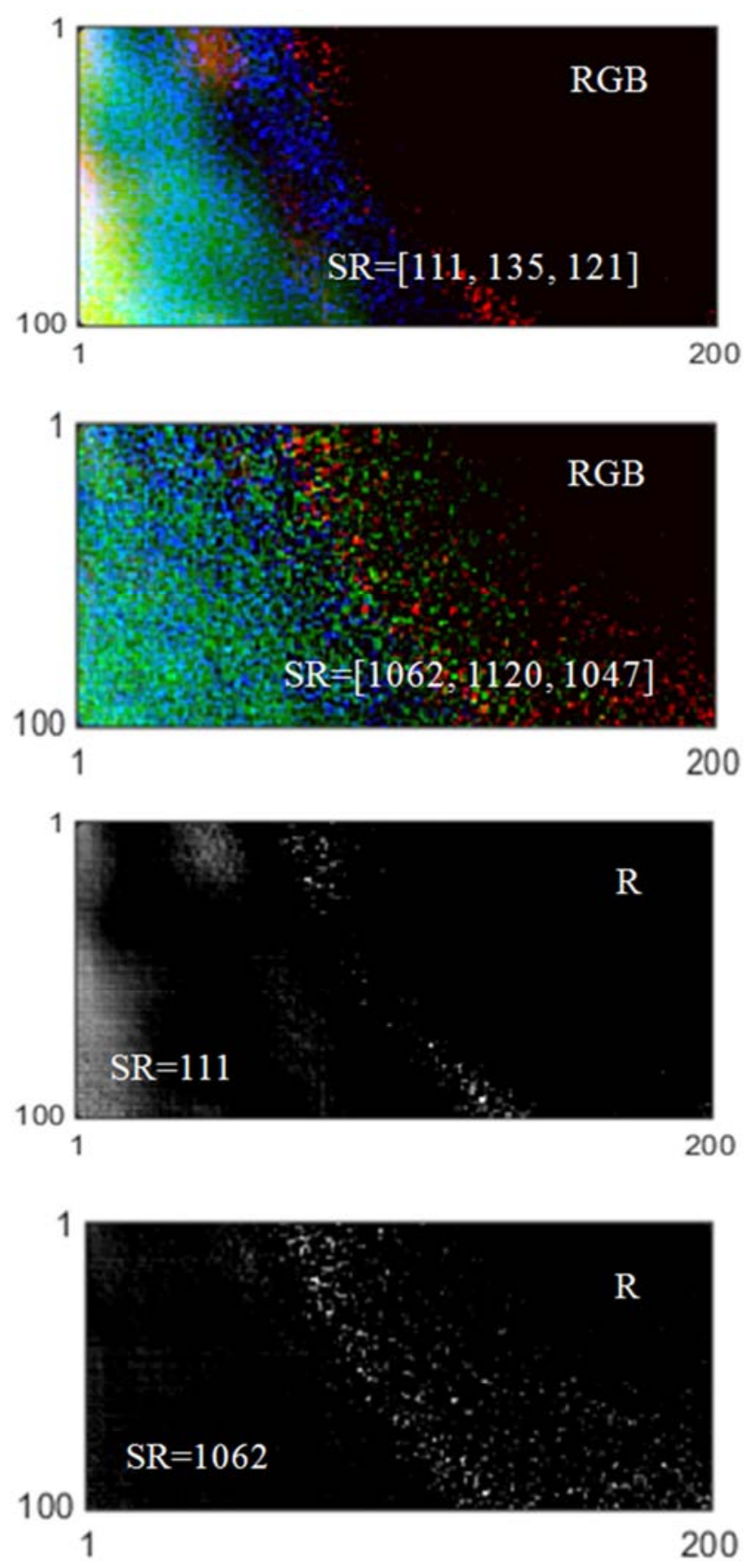

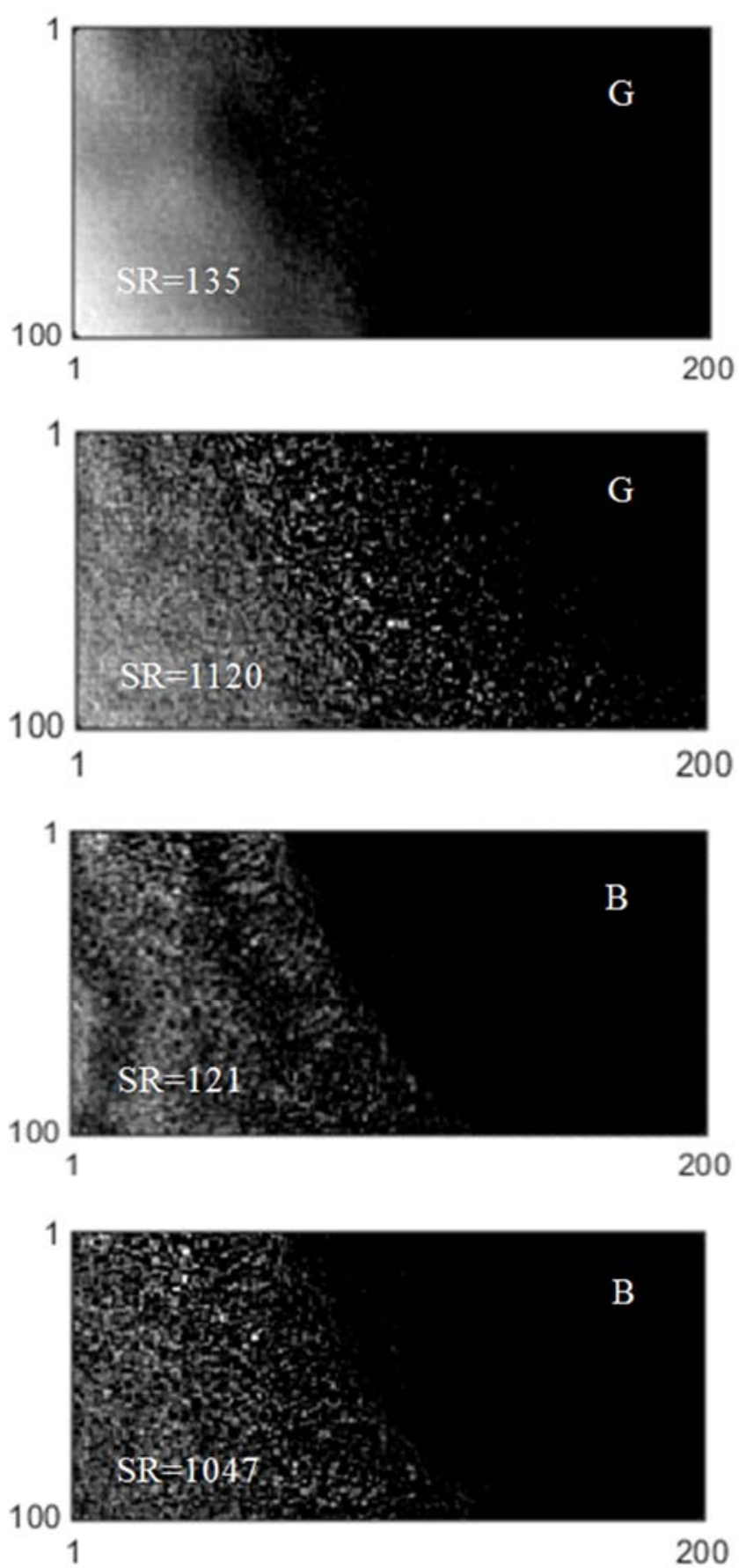

Figure 5. Full grid with the ste $=1$, with $D I=10^{4}$ and $5 * 10^{4}$.

In the next three section, we demonstrate the insides of the $\mathrm{MM}$ on three conditionality DI different occasions.

\subsection{Mantissa Errors, Maximum SR During Iz Inversion $\mathrm{AF}_{\mathrm{z}} \mathrm{O}_{\mathrm{G}}$}

According to the Theorem: $\operatorname{II}\left(z \mathrm{Z}^{*} \mathrm{O}\right)=1$, the reversibility follows with the accuracy Iz:

$\mathrm{zR}=\mathrm{R}=\mathrm{O}^{-1}, \mathrm{zR}^{*} \mathrm{O}=\mathrm{DK}$, MTF $\mathrm{M}(\mathrm{zR} * \mathrm{O})==1$, we have full Band Pass $\mathrm{M}(\mathrm{zR}) \mathrm{M}(\mathrm{O})==1, \mathrm{BP}=5132$ and we have the huge reaction to noise $\operatorname{Nor}(\mathrm{zR}) \sim 4.6^{*} 10^{10}$.
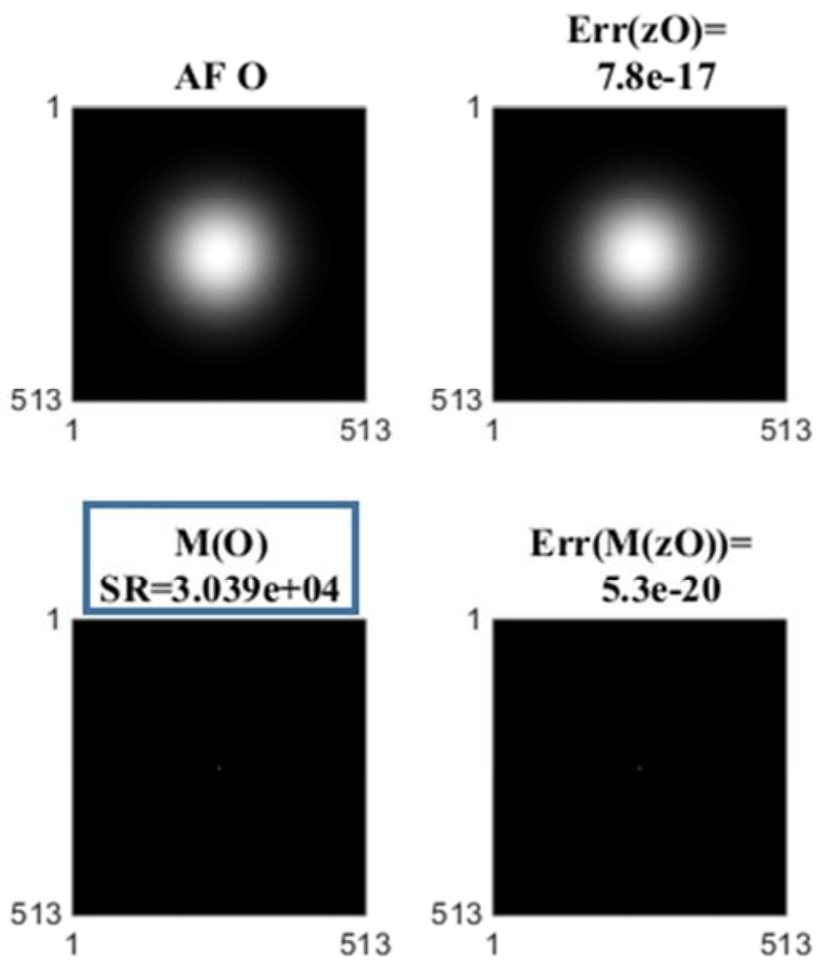

(a)
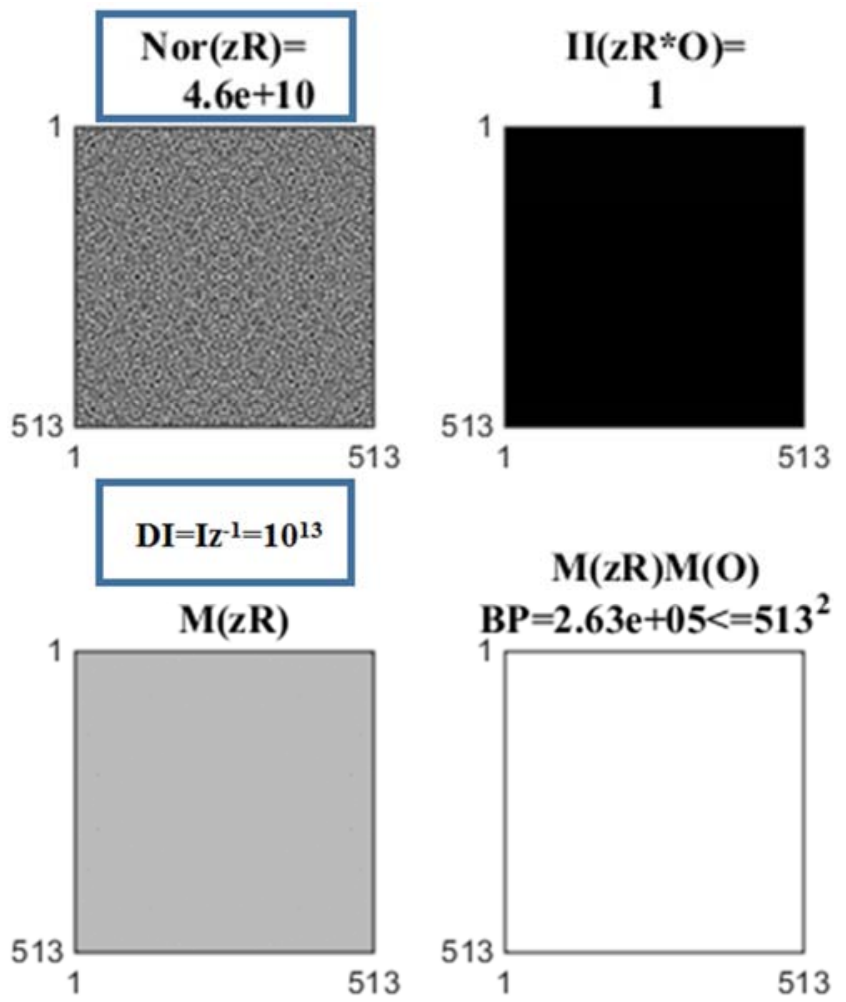

(b)

Figure 6. In (a-b) are Insides of Iz inversion (3-4), max $S R \sim 30000$ during Iz inversion $\mathrm{AF} z \mathrm{O}_{G}$.

\subsection{Conditionality in Adjustment of Inversion $\mathrm{AF} \mathrm{pO}_{G}$, $D I=2100$}

When setting the conditionality of the DI to 2100 , we lose 
in the reversibility of the $\mathrm{II}(\mathrm{pR} * \mathrm{O})=0.0019$, we get $\operatorname{Err}(\mathrm{pO})=2.8 \%$, we reset the response to noise till $\mathrm{DI} \sim \operatorname{Nor}(\mathrm{pR})=2100$ and we get the small value $\mathrm{SR} \sim 60$ like in the theatrical binoculars.
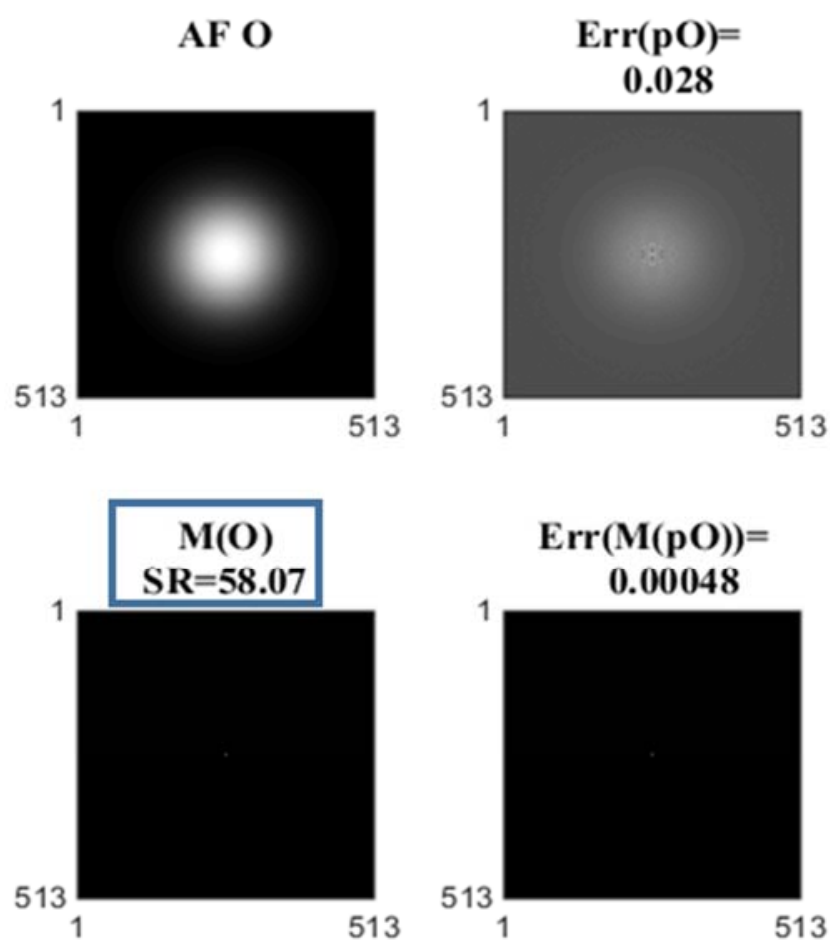

(a)
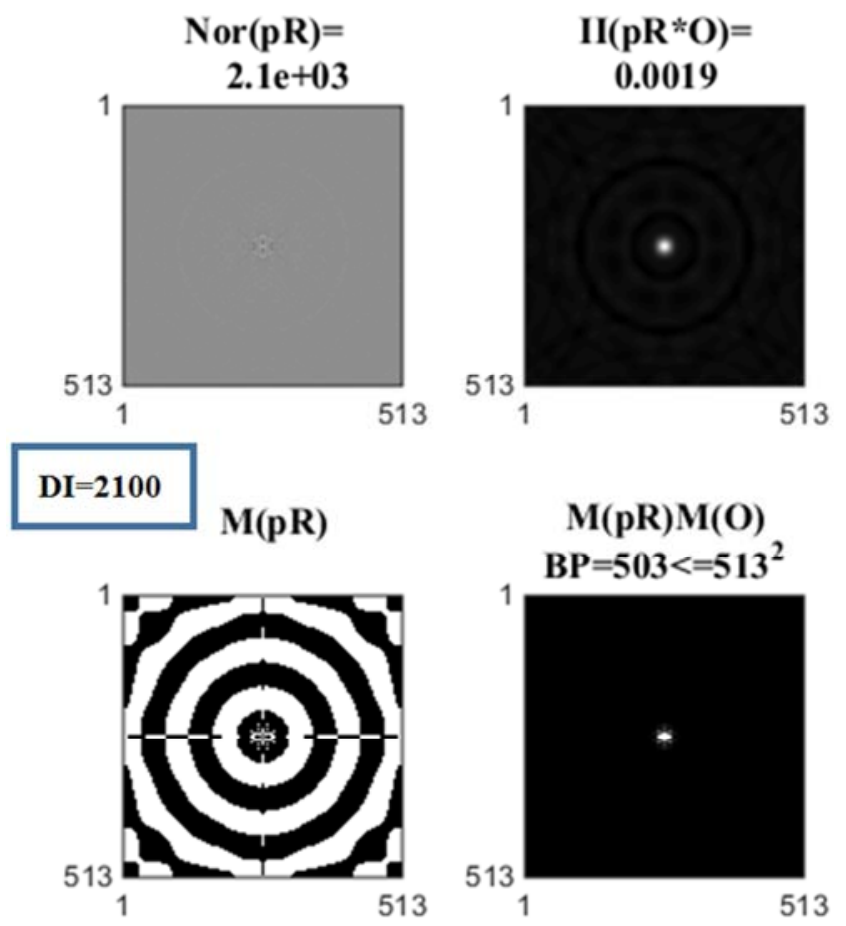

(b)

Figure 7. (a-b) are Insides of Conditional Inversion (1-2), conditionality in adjustment of inversion $A F p O_{G}, D I=2100$.

\subsection{Conditionality in Adjustment of Inversion $\mathrm{AF} p \mathrm{O}_{B}$, $D I=10^{5}$}
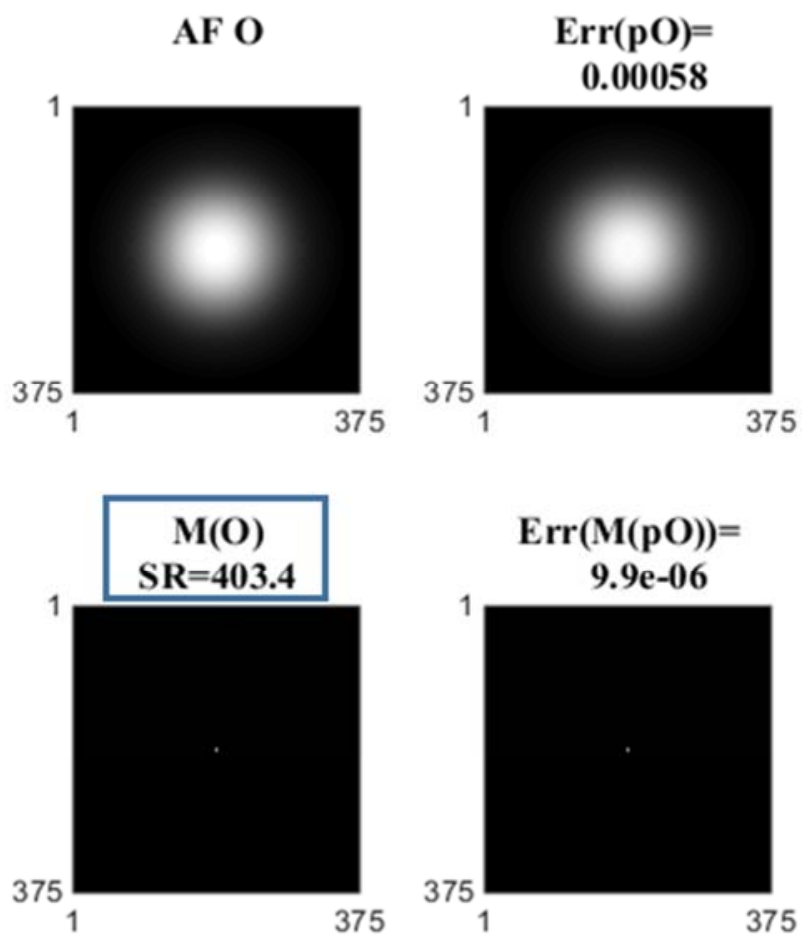

(a)
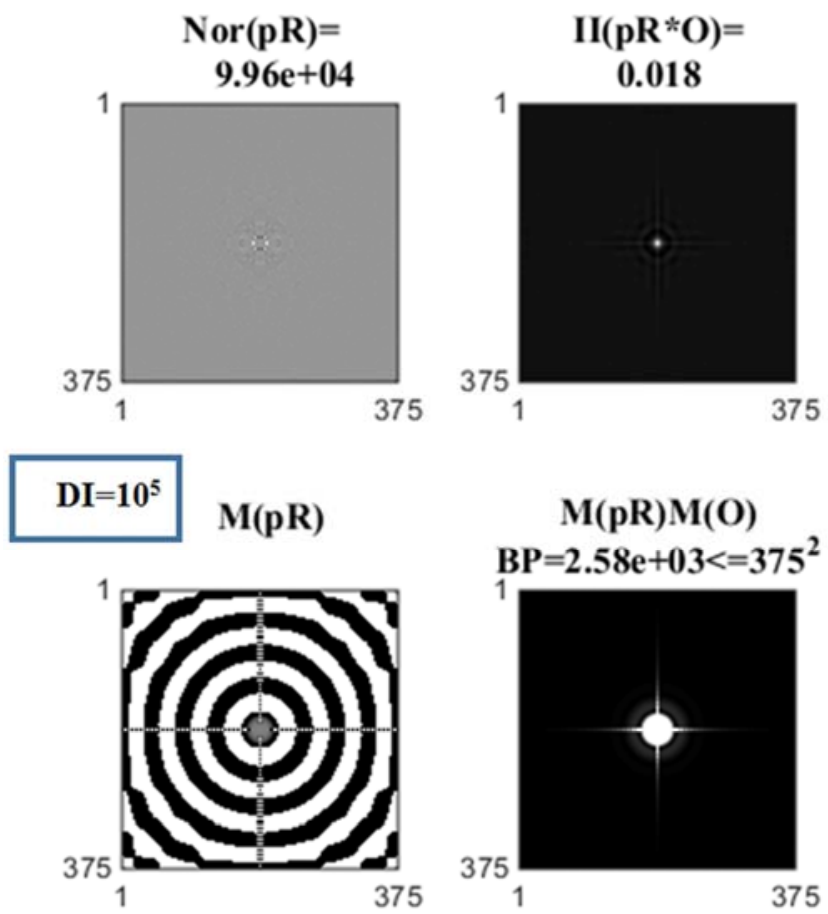

(b)

Figure 8. (a-b) are Insides of Conditional Inversion (1-2), Conditionality in adjustment of inversion $A F$ p $O_{B}, D I=10^{5}$.

When setting the conditionality of the DI to $10^{5}$, we lose in the reversibility of the $\mathrm{II}(\mathrm{pR} * \mathrm{O})=0.018$, we get $\operatorname{Err}(\mathrm{pO})=0.028$ we reset the response to noise till DI Nor $(\mathrm{pR})=10^{5}$ and we get the good value $S R \sim 400$ like in a children's microscope. 


\section{EHT Image Focusing in MM}

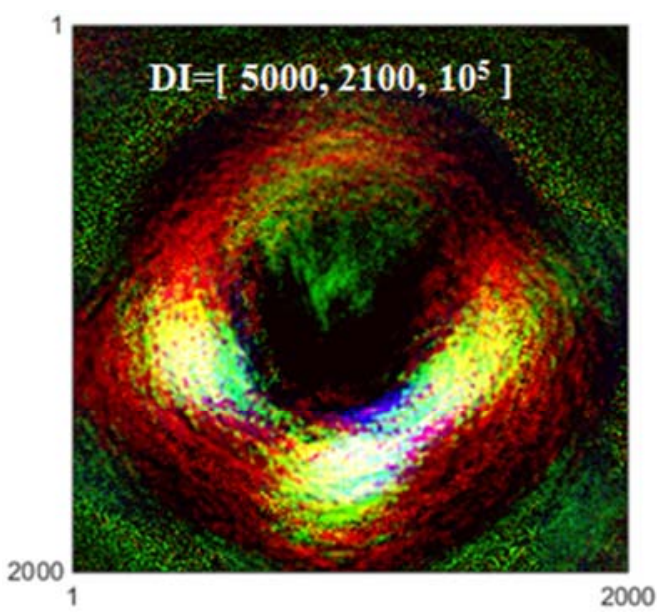

(a)

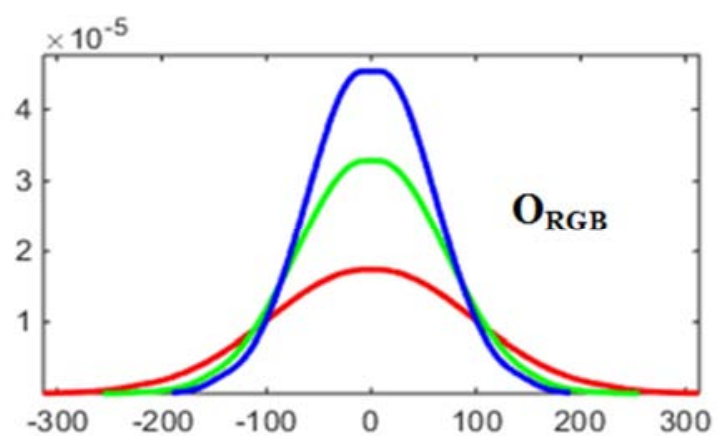

(b)

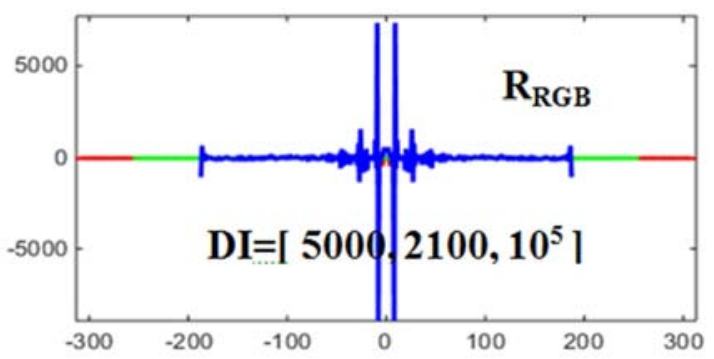

(c)

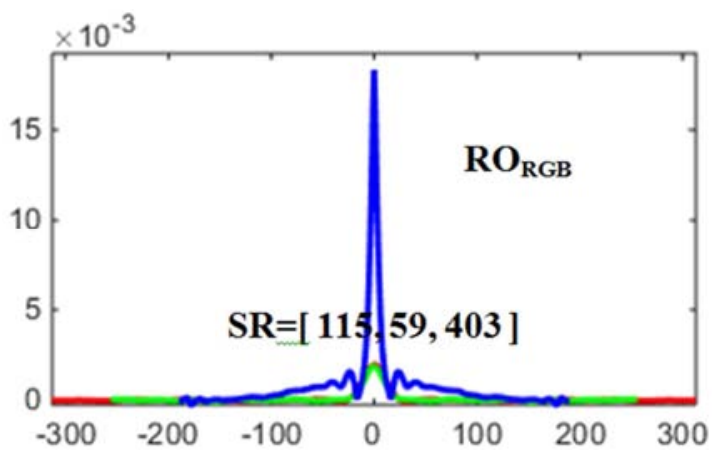

(d)

Figure 9. (a) - the CSR image, (b-d) - “cross sections” Reconstructed, Invers and Resolved AFs: $R O_{R G B}=\left\{R_{R} * O_{R}, R_{G} * O_{G}, R_{B} * O_{B}\right\}$.

The Event Horizon Telescope (EHT) [9] consists of 8 receiving synchronized (in time by atomic clock) antennas in three radio bands $\{\mathrm{R}, \mathrm{G}, \mathrm{B}\}$.

Professor Katerine L. Bouman first implemented the mathematical docking [12] of data from 8 telescopes, turning them into one EHT with a large aperture $(\sim 10000 \mathrm{~km})$ and received the first image of the Powehi $\mathrm{BH}[2,11]$, see in Figure 1(a).

Focusing in MM is regulated by DI conditionality from the range of values $[1,1 / \mathrm{min}|\mathrm{M}(\mathrm{O})|]$, that is, starting from the $\mathrm{AF} \mathrm{DK}=\mathrm{DK}^{-1}$ we pass $\mathrm{pR}=\mathrm{pO}^{-1}$ to the reversed $\mathrm{AF} \mathrm{zR}=\mathrm{ZO}^{-1}$.

Figure $12(b-c)$ shows the "cross-sections of the points" of which the images focused on conditionality at the output of the MM consist, see Figure 11 (a), Figure 1(a).
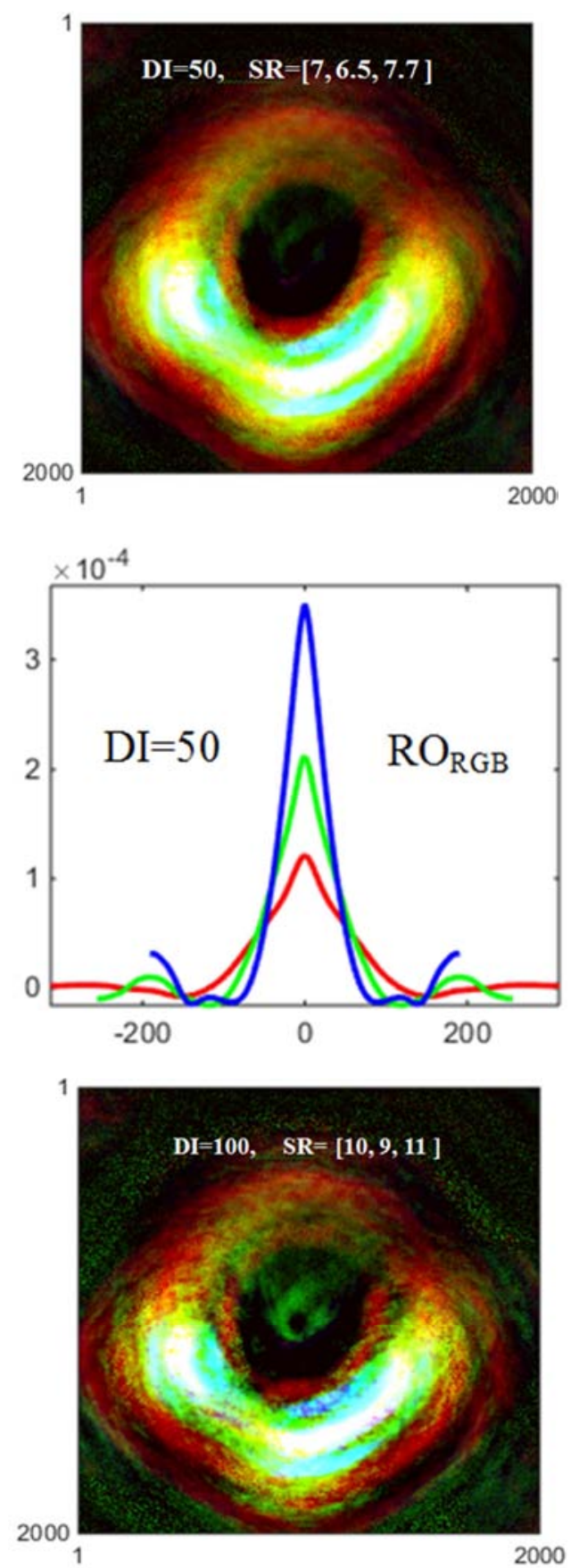

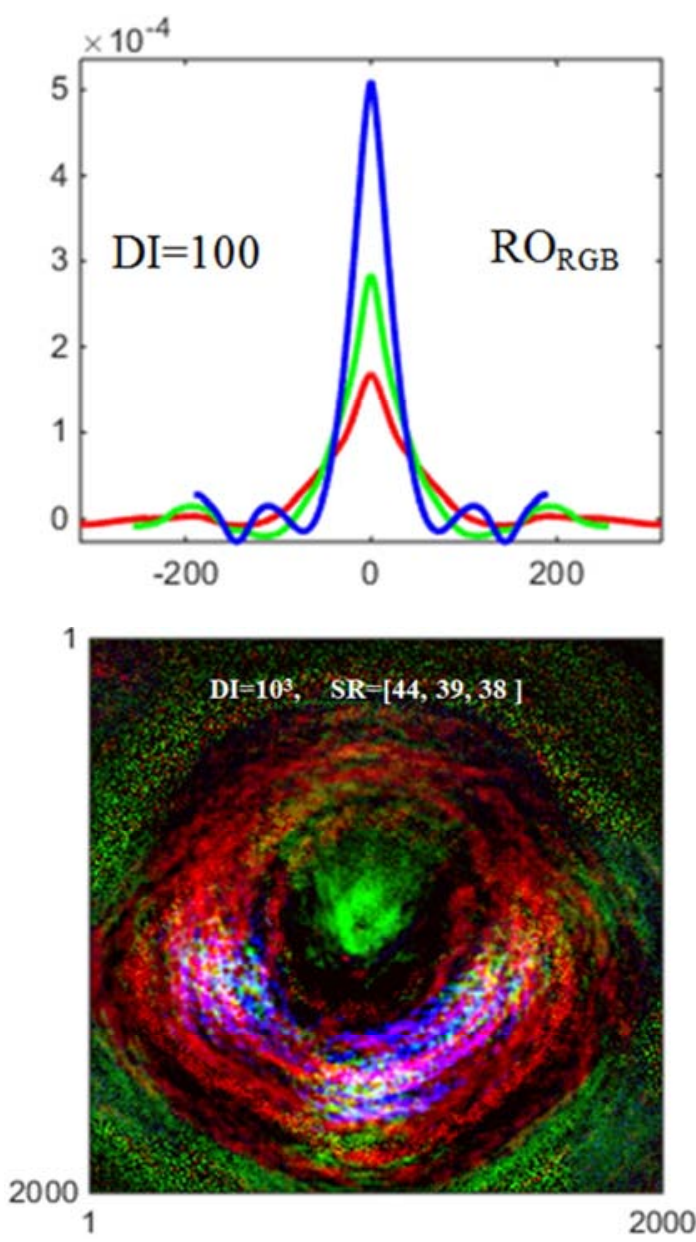

tomography, telescopes, in the manufacture of processors and memory, etc.

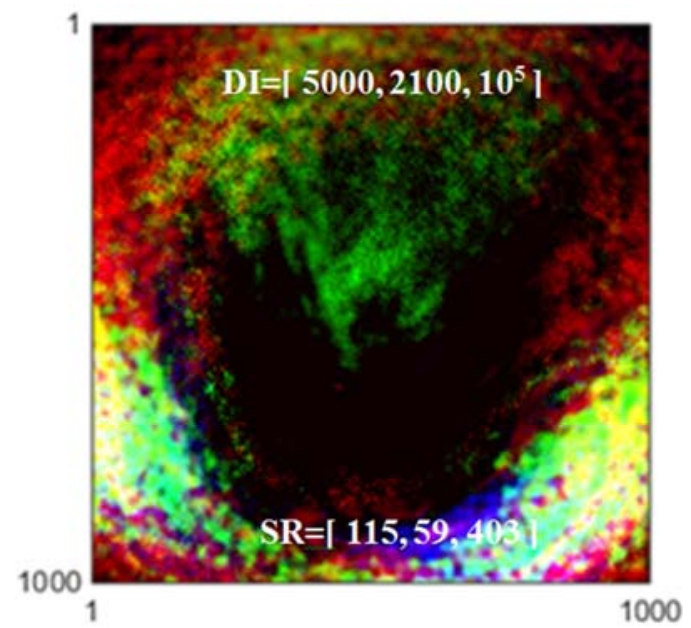

(a)

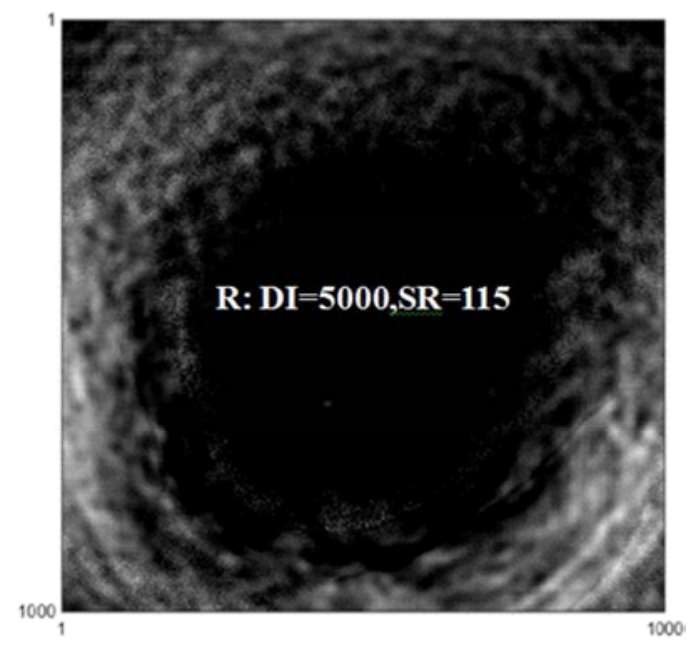

(b)

Figure 11. (a) - CSR image with the highlighted central part and in the $R$ channel (b).

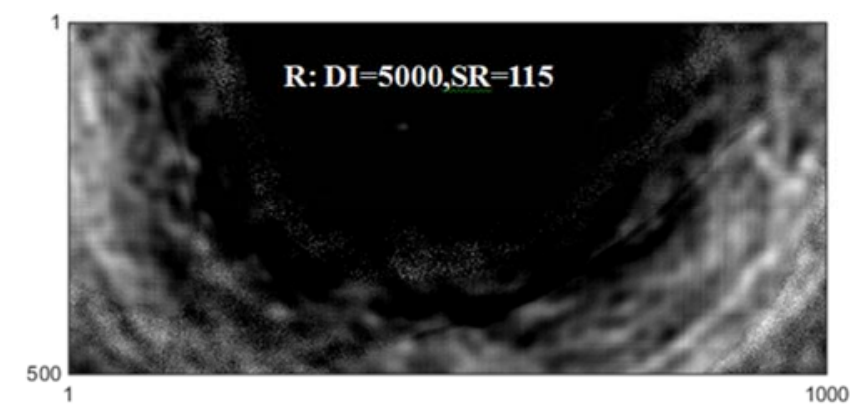

Figure 12. CSR fragment of the image on channel $R$.

In the CSR AF method, the resolved $\mathrm{RO}_{\mathrm{RGB}} \mathrm{AFs}$ are more "narrow-thin", compared to the original $\mathrm{O}_{\mathrm{RGB}} \mathrm{AFs}$; the super-resolution values SR (5). The obtained CSR image correspond to a virtual EHT with an aperture from the Earth to the Moon.

Modifications of the CSR AF methods [2-8] can be implemented in electron microscopy, in new radar technologies, in synthetic aperture radars, CT, MRT

On channel R, we observe the movement of the $\mathrm{BH}$ shadow in the direction of the arrow, a cluster of stars in front of the BH shadow, two streamline stellar current tubes and clusters of stars behind the BH, see Figure 13 below. 


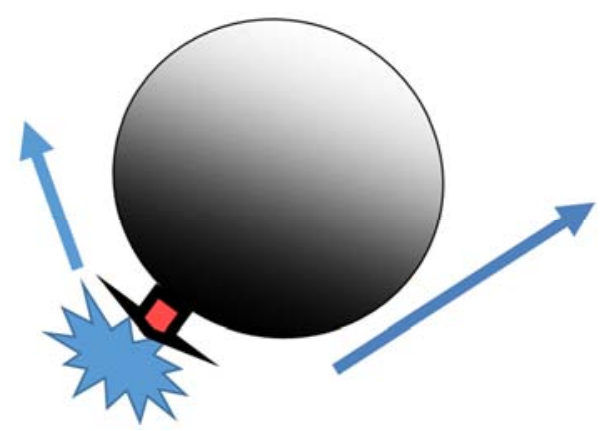

Figure 13. The BH moves to a cluster of stars, the BH is traversed through two tubes of star streams.

\section{Results in Collaboration EHT}

Below are the texts taken from the AIP messages, see [https://physicstoday.scitation.org/do/10.1063/PT.6.1.201904 $11 \mathrm{a} /$ full/].

The imaging efforts came to a head at the weeklong June 2018 meeting in Cambridge, Massachusetts. Over the first few days, the four groups met separately but then began to share details about the intricacies of their analyses. Finally, on 25 June, the entire EHT imaging team viewed all four images. They weren't identical, but they all shared a fundamental feature: a roughly 40 $\mu$ as photon ring surrounding an orb of darkness, the long-sought silhouette of a black hole. "It was a remarkable moment," says imaging team coleader Kazunori Akiyama of MIT

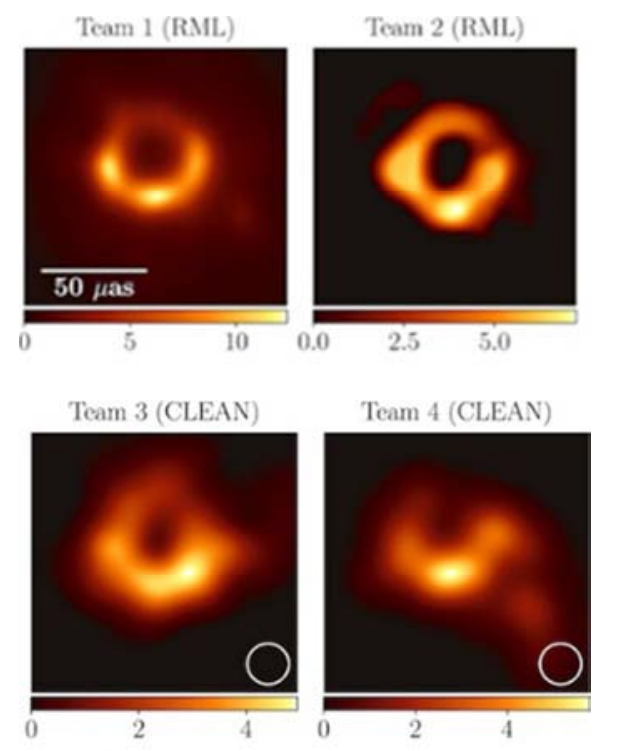

Figure 14. Despite taking different approaches to analyzing the petabytes of EHT data, four teams ended up with relatively similar images of M87*.

Not only did the images of the four groups agree with each other, but they also matched the output of models. Other members of the EHT team had developed General Relativistic Magneto Hydrodynamic Simulations (GRMHS) to first predict what EHT might see and then validate what it did see. The researchers ran thousands of simulations, each one with slightly different values for properties such as plasma temperature and the black hole's spin and magnetic flux.

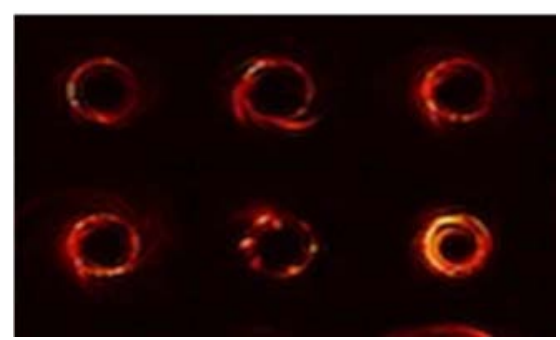

Figure 15. In the array of GRMHS images, the modeled black holes look similar to the measured one.

\section{2020 Nobel Laureates in Physics}

Roger Penrose, Reinhard Genzel, and Andrea Ghez are to be awarded the 2020 Nobel Prize in Physics for their theoretical and observational work on black holes, see [https://www.nobelprize.org/prizes/physics/2020/summary/].

Genzel (Max Planck Institute for Extraterrestrial Physics and the University of California, Berkeley) and Ghez (UCLA) each led a team that advanced the techniques of speckle imaging and adaptive optics to obviate atmospheric turbulence and analyze the motion of stars tightly orbiting Sagittarius A*, the radio source at the Milky Way's center.

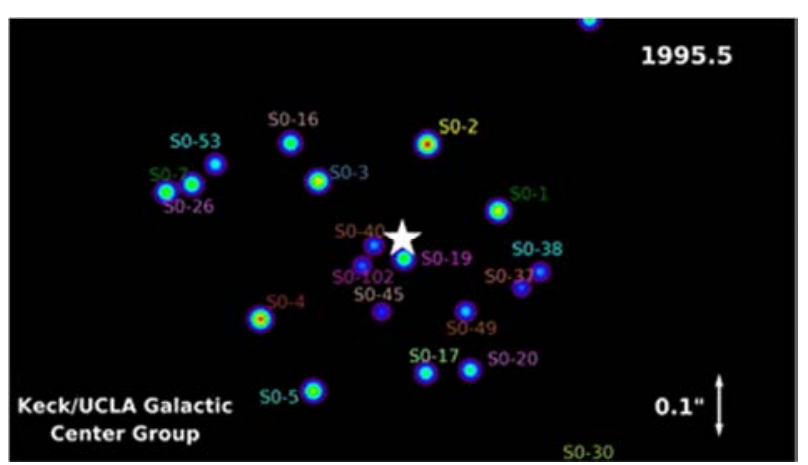

Figure 16. The researchers concluded that only a black hole, weighing in at about 4 million solar masses, could be responsible for the orbits they observed.

The decision of Swedish academics to award a prize for discoveries that are more theoretical in nature looks unusual.

\section{Discussions, AI for MM}

Our plans are to bring the SR value up to 2000-3000 times (like in a good optical microscope) on RGB channels and calculate all four frames of the surveys for April 5, 6 and 10, 11 April 2017, with control over the displacements of star clusters and, possibly, individual stars, as in Figures 15. Note that one eso frame for April 10 "weighs" 183.4 MB [2].

The results in this article were obtained by calculations on a laptop with a first generation Core i7 processor six years ago. One calculation option took approximately 3.5 minutes. Enumeration of a huge number of options is required on a powerful computer with an accelerator.

Of course, $\mathrm{AF} \mathrm{O}_{\mathrm{RGB}}$ reconstructions start with coarse grids like ste $=10,5[9,10]$. The most valuable thing in this work is the AF $\mathrm{O}_{\mathrm{RGB}}$ reconstructions (see Figures 3-5 and only then 
the SR images themselves (in Figures 10, 13, 14), in which point objects - stars are present.

Collaboration EHT is currently trying to verify the received data. In the first unsuccessful attempts to increase the resolution, see the six open access articles in [https://iopscience.iop.org/issue/2041-8205/875/1], point objects - star clusters were not identified. Even the original issue of AF or EHT Antenna Pattern estimation is not addressed.

Note that in the schematic drawing (Figure 14) BH "enters" the stellar matter, there is no vortex (as in Figure 15), we see reactive spitting in the G channel, see Figure 9(a).

Note that based on SR images, the number of GRMHS; see Figure 15 will be significantly reduced down to one model, as it should be in a physical experiment.

At various scientific sites in Russia with the presence of foreign scientists, a wide range of problems of the creation and use of Artificial Intelligence (AI) are currently being discussed.

Below we give the headlines of discussions about AI close to this work.

AI in Math Physics Modeling for Controlling Local Phenomena and Objects, two Projects:

P1: AI for Mathematical Microscope

P2: AI in Local Phenomena and Object Recognition

$\mathrm{P} 1$ : AI for MM is intended for the Intelligent Analysis (IA) of data on objects observed through measuring devices whose Apparatus Functions (AF) are not defined, are known with errors and even when they are precisely known.

The estimation-reconstruction of the Reversible Apparatus Function $\mathrm{R}=\mathrm{A}^{-1}$ is realized by point objects in the Super-Resolved images by a human operator after viewing the SR images "manually". We propose to create AI for the automatic solution of the SR problem.

P2: $\mathrm{AI}$ in $\mathrm{OR}$ is intended for the analysis of parameters of local phenomena and objects for their recognition in images by using methods of Gradient Morphology (GM) [13, 14].

GM methods are designed to accurately determine the parameters of such objects as vortices in space images or in the BH images, Traffic Signs, Subtle Features of the Face, etc. Recognition is realized by the values of the parameters. We associate such complex programs with AI development.

We believe that the indicated problems $\mathrm{P} 1$ and $\mathrm{P} 2$ on the creation of $\mathrm{AI}$ in the next 2-3 years will be implemented.

\section{Acknowledgements}

Congratulations to all 347 awarded the Breakthrough 2020 Prize in the field of fundamental physics of joint work on the EHT, see: https://breakthroughprize.org/News/54.

Special acknowledgments to Professor Katerine L. Bouman of the California Institute of Technology [12]. High data quality [2] allowed us to implement MM for EHT.

\section{References}

[1] A. N. Tikhonov, M. V. Ufimtsev "Statistical processing of experimental results", ed. Moscow University Press, 1988 (in Russian).

[2] https://www.eso.org/public/images/eso1907a/.

[3] E. N. Terentiev, N. E. Terentyev//Izvestia RAN, PHYSICAL SERIES, 2015, volume 79, № 12, c. 1633-1637 (in Russian).

[4] Terentiev, E. N. and Terentiev, N. E.//ISSN 1062-8738, Bulletin of the Russian Academy of Science. Physics, 2015, Vol. 79, No 12, pp. 1427-1431, DOI $10.3103 /$ S1062873815120229.

[5] E. N. Terentiev, N. E. Terentyev, Yu. A. Pirogov, I. I. Farshakova//SCIENTIFIC NOTES OF THE PHYSICAL FACULTY, 9 p., №6, 1761005,(2017) (in Russian).

[6] Terentiev, E. N., Terentiev, N. E., Farshakova, I. I.//DOI: 10.1007/978-3-319-77788-7_19.

[7] E. N. Terentiev, N. Shilin-Terentyev//doi.org/10.1007/978-3-030-11533-3_44.

[8] E. N. Terentiev, I. I. Farshakova, I. N. Prikhodko, N. E. Shilin-Terentyev//doi: 10.11648/j.sjams.20190705.12, ISSN: 2376-9491 (Print); ISSN: 2376-9513 (Online).

[9] E. N. Terentiev, I. I. Farshakova, N. E. Shilin-Terentyev//http://www.sciencepublishinggroup.com/journa 1/paperinfo?journalid=301\&doi=10.11648/j.ajaa.20190703.11.

[10] E. N. Terentiev, I. N. Prikhodko and I. I. Farshakova//Concept of mathematical microscope, AIP Conference Proceedings 2171, 110010 (2019); https://doi.org/10.1063/1.5133244.

[11] The Event Horizon Telescope Collaboration, First M87 Event Horizon Telescope Results. I. The Shadow of the Supermassive Black Hole, The Astrophysical Journal Letters, 875: L1 (17pp), 2019 April 10, https://doi.org/10.3847/2041-8213/ab0ec7.

[12] Bouman, Katherine L.; Johnson, Michael D.; Zoran, Daniel; Fish, Vincent L.; Doeleman, Sheperd S.; Freeman, William T. (2016). "Computational Imaging for VLBI Image Reconstruction": 913-922. arXiv: 1512.01413, doi: 10.1109/CVPR.2016.105, hdl: 1721.1/103077. Cite journal requires journal $=($ help$)$.

[13] E. N. Terentiev, I. N. Prikhodko and I. I. Farshakova//Problems of accurate localization objects in imagers, AIP Conference Proceedings 2171, $110009 \quad$ (2019); https://doi.org/10.1063/1.5133243.

[14] E. N. Terentiev, I. N. Prikhodko, and I. I. Farshakova//Applications of finite dimensional sampling theories, AIP Conference Proceedings 2195, 020019 (2019); https://doi.org/10.1063/1.5140119. 